\title{
Urinary Tract and Vaginal Infections caused by Group B Streptococcus and the Macrolide-Inducible Resistance to Clindamycin in Non-pregnant Females
}

\author{
Mona F. Foad* \\ Department of Clinical Pathology, Faculty of Medicine, Mansoura University, Egypt \\ *Corresponding author
}

\begin{tabular}{|c|c|}
\hline & \multirow{9}{*}{ 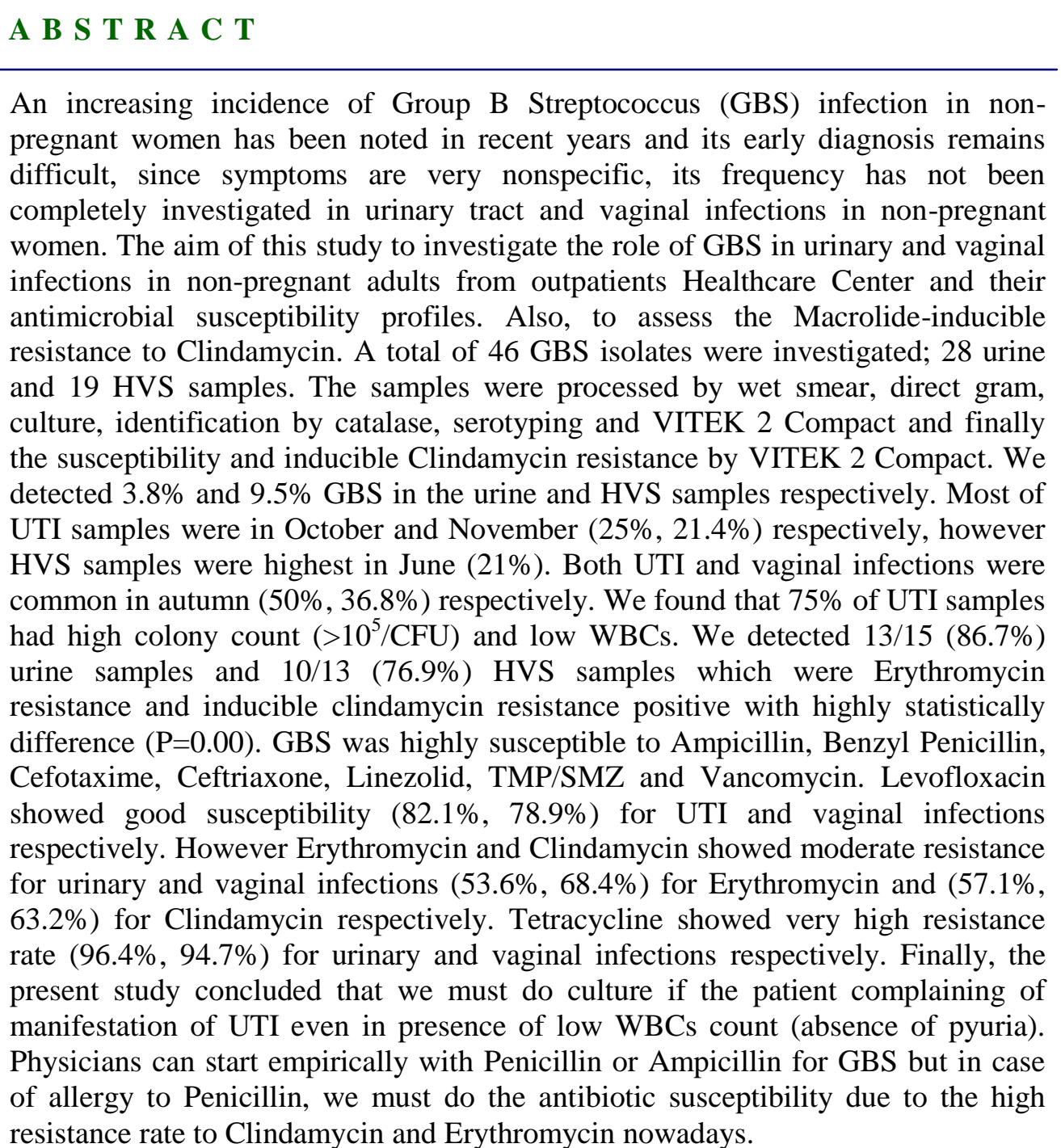 } \\
\hline & \\
\hline & \\
\hline & \\
\hline & \\
\hline & \\
\hline 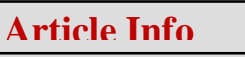 & \\
\hline & \\
\hline & \\
\hline
\end{tabular}




\section{Introduction}

Streptococcus agalactiae, also known as Group B Streptococcus (GBS) is considered a common commensal of the female urogenital tract and rectum (Schuchat, 1999). Its importance is referred to severe neonatal pathologies by perinatal transmission from women to new-born (Lambiase et al., 2012). Bacterial colonization is seen in about $10 \%$ of nonpregnant women and $25 \%$ of pregnant individuals (Mandell et al., 2000).

The number of cases of invasive infection caused by $S$. agalactiae in non-pregnant adults is increasing (Huang et al., 2006; Matsubara and Yamamoto, 2009) especially in patients that had underlying medical conditions. The mortality rate range is 3$47 \%$ being highest in elderly patients with underlying medical conditions (Phares et al., 2008).

Group B Streptococcus is a causative agent of urinary tract infections (UTI). The spectrum of GBS UTI includes asymptomatic bacteriuria (ABU), cystitis, pyelonephritis, urethritis and urosepsis (Edwards and Baker, 2005; Mckenna et al., 2003).Clinically, UTI caused by GBS in many times may be indistinguishable from those caused by other organisms. However many studies suggest that microbiological and clinical features may be different depending upon the causative agent (Edwards et al., 2005; Bliss et al., 2002). Various rate of GBS UTI rate have been reported in pregnant and non-pregnant adults (Nolla et al., 2003). GBS UTI rate have been different from $1 \%$ to up to one third and even more in these studies (Falagas et al., 2006; Toumi et al., 2006).

Group B Streptococcus colonization of the urinary tract in women most likely occurs by an ascending route from the vagina, where
GBS can persist asymptomatically. The overall prevalence of GBS UTI in the adult population remains unclear (Ulett et al., 2009).

Penicillin $G$ and Ampicillin are the antibiotics of choice for intrapartum prophylaxis and treatment of invasive infections, while Clindamycin is the recommended agent for patients who are allergic to beta-lactams (Matteson et al., 2008). However, although S. agalactiae remains almost always susceptible to Penicillins, there is a significant and rising resistance to macrolides and lincosamides (Gygax et al., 2006).

Two major resistance mechanisms to macrolides and lincosamides have been reported: alteration of the antibiotic target site (encoded by erm genes) and active drug efflux pump (encoded $b$ mef gene) (Domelier et al., 2008). Macrolides is usually mediated by the erm and mef genes, erm-mediated resistance includes macrolidlincomycin-streptogramin b-inducible (iMLSB) and constitutive (cMLSb) phenotypes. Both MLSb phenotypes are Eresistant and clindamycin resistant $\left(\mathrm{CC}^{\mathrm{R}}\right)$ but iMLSb requires induction to show $\mathrm{CC}^{\mathrm{R}}$, mef ( $\mathrm{M}$ phenotype) are $\mathrm{E}^{\mathrm{R}}$ and $\mathrm{CC}$ susceptible $\left(\mathrm{CC}^{\mathrm{S}}\right)$ (Gosnell et al., 2005).

There are only few and limited studies to determine the role of GBS in UTI and vaginal infections in non-pregnant adults. The present study was conducted to investigate the role of GBS in both infections in non-pregnant adults from outpatients Healthcare Center and their antimicrobial susceptibility profiles and to assess the Macrolide-inducible resistance to Clindamycin.

\section{Materials and Methods}

This study was conducted on female patients visited a primary healthcare out-patient 
clinic in UAE. The first non-pregnant group $(n=28)$ complaining of at least one symptom of urinary tract infection that included dysuria, increased urinary frequency and/or urgency, fever of $>38^{\circ} \mathrm{C}$, flank and/or lumbar tenderness. The other non-pregnant group $(n=19)$ included patients complaining of vaginal infection in the form of discharge in the period from January 2015 to December 2015.

Morning mid-stream urine samples were collected in sterilized, leak-proofcontainers before the start of antibiotic. The samples were cultured on blood, MacConkey agar by calibrated loop(1ul) and incubated $24 \mathrm{hs}$ in $37^{\circ} \mathrm{C}$. Urine was examined microscopically and chemically by iQ200 $2^{\text {nd }}$ generation Automated Urine Microscopy AnalyzerHVL (High volume laboratories) which can process 60 samples/h (Iris Diagnostic. Chatsworth, CA).

\section{Iris $\mathrm{iQ200} 2^{\text {nd }}$ generation}

It is an in-vitro diagnostic use device composed of the iQ200 Automated Urine Microscopy Analyzer, connected physically and electronically to the AUTION MAX TM AX- 4280 Automated Urine Chemistry Analyzer and a workstation. It is a walkaway system that uses flow imaging analysis technology and Auto-Particle Recognition (APR, Iris Diagnostic) software to classify particles based on multiple parameters. Images are stored and can be viewed on the workstation screen, thereby eliminating the need for manual microscopy.

High vaginal swab (HVS) samples were cultured on blood, MacConkey, Chocolate agar in $\left(5-10 \% \quad \mathrm{CO}_{2}\right)$ for $24 \mathrm{hs}$ and Sabouraud's agar for $48 \mathrm{hrs}$ in $37^{\circ} \mathrm{C}$. Wet smears were examined microscopically for Trichomonas, WBCs, RBCs, epithelial cells or yeast. Direct film stained with Gram stain was examined for Clue cell, Gram Negative diplococci or yeast.

Identification of GBS was based on the recognition of beta-hemolytic colonies on sheep blood agar, Gram-positive cocci on Gram stain, absence of catalase production and latex agglutination with group-specific antiserum (BioMerieux).

\section{Slidex Strepto plus}

It is a latex agglutination test done for Streptococci bacteria of lancefield group A, $\mathrm{B}, \mathrm{C}, \mathrm{D}, \mathrm{F}$ and $\mathrm{G}$. It is latex particles conjugated to group specific antisera will bind to the corresponding antigen to result in visible clumping of the latex particles. Most beta hemolytic Streptococci possess group specific antigens that can be extracted and identified with group specific antisera.

After growth, the isolated colonies of Streptococci are removed and placed in a tube containing the extraction enzyme. The group specific antigen is enzymatically extracted from the streptococcal cell wall. Antigen in the extract is identified using latex particles sensitized with group specific anti-streptococcal antibody. Visible aggregates form in the specific latex particle suspension which reacts with the extracted antigen. The latex will remain in suspension if the antigen is not present in the extract.

\section{Methods}

For the preparation of the extract: transfer $0.4 \mathrm{ml}$ of the extraction enzyme into test tube, then pick 3-5 typical colonies according to their size and emulsify them in the extraction enzyme.

Mix using a vortex-type mixer and incubate for ten min at $37^{\circ} \mathrm{C}$, then dispense 1 drop of each latex reagent into the corresponding 
reaction fields (A,B,C,D,F and $\mathrm{G}$ ). Then dispense $15 \mathrm{ml}$ of the extract beside each together and spread over the whole surface of the field. Rotate the card gently for a maximum of 2 min and read under normal lighting.

A positive result is indicated by the development of an agglutination pattern showing clearly visible clumping of the latex particles in less than $2 \mathrm{~min}$.

A negative result is indicated when agglutination doesn't occur. Homogenous suspension or very fine granular appearance.

The result is uninterruptible if agglutination is observed in several of the latex suspension. This may indicate a polyagglutinating strain or a mixture of strains. In this case, reperform the isolation and test.

After serotyping, we did Automated identification and susceptibility on Vitek 2 Compact (BioMerieux, France), machine used for fast (5-8 hs) and accurate microbial identification.

\section{VITEK 2 compact machine}

It includes an expanded identification database, andreads every $15 \mathrm{~min}$ for greater speed in identification.

It uses Advanced Colorimetry ${ }^{\mathrm{TM}}$

We use Gram +ve identification and AST ST01 Streptococcus Susceptibility card; the panel includes: Ampicillin (AM), Benzylpenicillin (P), Cefotaxime (CTX), Ceftriaxone (CRO), Clindamycin (CM), Erythromycin (E), Levofloxacin (LEV), Linezolid (LNZ), Tetracycline (TE), Timethoprim/ Sulfamethoxazole (SXT) and Vancomycin (VA).
VITEK 2 compact test for inducible Clindamycin Resistance (ICR): for Strept. Pyogen and Strept. Aglactiae

Clindamycin $\quad(\mathrm{CM}) \quad 0.5 \quad \mathrm{ug} / \mathrm{ml}$, Clindamycin/Erythromycin 0.25/0.5 ug/ml.

This test is quantitative growth based detection algorithm using predetermined growth thresholds.

\section{Statistical analysis}

Data were analyzed using SPSS (Statistical Package for Social Science) version 19. Qualitative data was presented as number and percentage. Quantitative data was presented as mean and standard deviation. The Chi-square was used to compare between variables of qualitative data. The $\mathrm{P}$ value of $<0.05$ indicates a significant difference while $\mathrm{P}$ value of $<0.001$ indicates a highly significant difference.

\section{Result and Discussion}

Of the 745 urine samples collected from non-pregnant females complaining of UTI; $549(73.7 \%)$ displayed no growth while 196 (26.3\%) were giving growth; from which 28 $(14.3 \%)$ were GBS, yielding a prevalence of $3.8 \%$ in total urine samples from nonpregnant females.

Of the 199 HVS samples from non-pregnant females complaining of vaginal infection; $122(61.3 \%)$ were giving no isolated pathogenic organism and 77 (38.7\%) showed pathogenic growth; from which 19 $(24.7 \%)$ were GBS, yielding a prevalence of $9.5 \%$ in total HVS from non-pregnant females. There were four patients with the same vaginal and urinary infection with GBS infection. 
Demographic data and microscopic examination of the urinary and vaginal samples were shown in table (1). A total number of 47 (28 urinary and 19 HVS) isolates of GBS were recovered out of 944 $(4.98 \%)$ samples collected from female patients complaining of manifestations of infection. There was insignificant difference between both groups of UTI and HVS samples as regard age, WBCs, RBCs and pus ( $\geq 10 \mathrm{WBCs} / \mathrm{HPF})$ counts.

Frequency of GBS in different months were shown in table (2). Most of UTI positive samples were detected in October and November $(25 \%, 21.4 \%)$, while HVS positive samples were highest in June (21\%).

Seasonal variation in each group was shown in table (3). Both UTI and vaginal infections were common in autumn $(50 \%, 36.8 \%)$ but without statistically significant difference.

Table (4) showed the frequency of colony count of positive cultures in UTI with WBCs/HPF. We found 21/28 (75\%) with high colony count and with low WBCs count $(<10 / \mathrm{HPF})$. However, we found only $7 / 28(25 \%)$ with high colony count and high WBCs count $(\geq 10 / \mathrm{HPF})$ with statistically insignificant difference $(\mathrm{P}=0.2)$.

We detected thirteen urine samples showing Erythromycin resistance(13/15) (86.7\%) and inducible Clindamycin positive; and clindamycin become resistant with highly statistically difference $(\mathrm{P}=0.000)$. However, ten HVS samples were Erythromycin resistant (10/13) (76.9\%) and inducible clindamycin positive; clindamycin become resistant with highly statistically significant difference $(\mathrm{P}=0.001)$.

Clindamycin resistant was detected in $(3 / 16)$ (18.8\%) urine samples and (2/12) (16.7\%) HVS with negative inducible clindamycin with highly statistically significant difference $(\mathrm{P}=0.000)$ (Table 5).

GBS was highly susceptible to Ampicillin, Benzyl Penicillin, Cefotaxime, Ceftriaxone, Linezolid, TMP/SMZ and Vancomycin. Levofloxacin showed good susceptibility for UTI (82.1\%) and for vaginal infection $(78.9 \%)$. However urinary and vaginal infections showed high resistance to Erythromycin $(53.6 \%, 68.4 \%)$ and for Clindamycin $(57.1 \%, 63.2 \%)$ respectively. The highest resistance was shown for Tetracycline $(96.4 \%, 94.7 \%)$ respectively (Table 6).

Streptococcus agalactiaeis generally known to cause invasive infection in pregnant women and neonates since it commonly colonizes the vaginal and gastrointestinal tracts of healthy women (Farley et al., 1993). However, infection in non-pregnant adults has been increasingly reported worldwide (Falagas et al., 2006; Huang et al., 2006; Matsubara and Yamamoto, 2009; Phares et al., 2008). This study showed that positive urine culture for GBS was found in $3.8 \%$ of all non-pregnant females subjects and $14.3 \%$ in females with positive culture.Shayanfar et al.,2012; Munoz et al., 1992; Ulett et al., 2009 reported lower prevalence of GBS $(1.8 \%, 2 \%, 1.1 \%)$ respectively. Rahbar et al., 2012 was in agreement with our results who detected the frequency rate of GBS was $4.22 \%$ among female patients engaging UTI.

GBS was detected in $9.5 \%$ of the total HVS from non-pregnant females, Lambiase et al., 2012 detected higher prevalence 879/2156 $(40.8 \%)$ from vaginal and recto-vaginal swabs that were obtained from pregnant and non-pregnant women, a 4-year study from 2005-2008.

The highest rates of cultures positive for GBS were seen in October and November 
$(25 \%, 21.4 \%)$ respectively for UTI and June (21\%) for vaginal infection. Both types of infections were common in autumn. Shayanfar et al., 2012 found that the highest rates of culture positive for GBS were seen in December and January (11.4\% for each). Chaiwarith et al., 2011 observed that the number of Strept. aglactiae was higher in September (autumn) and they did not know the explanation for this finding but there is controversy about the seasonal variation due to geographical distribution and variation in seasons.

The mean WBCs in urine samples in our study was $85.9 \pm 315$ (ranging from 1-1600), most of the study subjects $(75 \%)$ showed WBCs count <10/HPF and $(25 \%)$ cases > $10 / \mathrm{HPF}$. The mean WBCs reported by Shayanfar et al., 2012 was very low $6.6 \pm 10.1$ (ranging from 0 to 50) but they were in agreement with our study that the majority of the studied subjects $(83.7 \%)$ showed WBCs <10/HPF and only $16.3 \%$ with WBCs>10/HPF.

Our study showed that $18 / 28(64.3 \%)$ of the patients had a colony count of $>10^{5}$ and $10 / 28(35.7 \%)$ had a colony count $10^{4}$ $10^{5}$.This was in agreement with a study by Munoz et al., 1992, the GBS colony count > $10^{5}$ in urine culture of $63 \%$ of the subjects, however Shayanfar et al.,2012 showed that only $10 \%$ of patients had a colony count of $>10^{5}$. The cause of this difference could be attributed to the study population.

We detected 13/15 (86.7\%) urine samples and 10/13 (76.9\%) HVS which were Erythromycin resistant and inducible clindamycin resistance positive with highly statistically difference $(\mathrm{P}=0.00)$. Gosnell et $a l ., 2005$ found lower percentage than our results of Erythromycin resistance and inducible clindamycin resistance positive 37/64 (57.8\%). Also, Browling et al., 2010 founded Erythromycin resistance and positive inducible clindamycin in 16/32 $(50 \%)$ of GBS and in $46 / 100(46 \%)$ of total Beta-hemolytic streptococcus isolates.

Erythromycin and Clindamycin in our study showed moderate resistance for urinary and vaginal infections $(53.6 \%, 68.4 \%)$ for Erythromycin and $(57.1 \%, 63.2 \%)$ for Clindamycin respectively and almost all Clindamycin resistant strains were also resistant to Erythromycin except one case. There are diversity of results, some studies showed lower resistance. Woods et al., 2015 found that $32 \%$ of GBS isolates were resistant to Erythromycin and $15 \%$ were resistant to Clindamycin and $99 \%$ of Clindamycin resistant strains were also resistant to Erythromycin. Also, Ulett et al., 2009 found resistance to Erythromycin $(39.5 \%)$ and $26.4 \%$ for Clindamycin.

Florindo et al., 2014 studied the increasing rate of resistance from 2006 to 2012, GBS isolates that were resistant to Erythromycin ranged from 14\% in 2006 to $23 \%$ in 2011, whereas the \% of GBS isolates with resistance to Clindamycin ranged from $6 \%$ in 2009 to $18 \%$ in 2012.Rahbar et al., 2012 were in agreement with our results, they reported resistance rate to Erythromycin and Clindamycin $(70.5 \%, 66.1 \%)$ respectively to GBS. Other studies showed higher resistance rate, Lambiase et al., found that the occurrence of Macrolide and Clindamycin resistance was $16.5 \%$ in 2005 increasing up to $69.9 \%$ in 2008 . Also, Tazi et al., 2007 reported $73.6 \%$ resistance to Erythromycin.

In our study, Tetracycline showed very high resistance rate $(96.4 \%, 94.7 \%)$ respectively for urinary and vaginal infections. Mostly all the studies showed the high resistance rate to Tetracycline (Lambiase et al, 2012; Florindo et al., 2014; Tazi et al., 2007). All 
GBS resistant to Erthromycin, also resistant to Tetracycline which could be expected considering a putative horizontal gene transfer event involving the same conjugative transposon carrying both genetic resistance determinants (Gherardi et al., 2007).There are two main mechanisms of resistance to Tetracycline in Streptococci; efflux by proton antiporters and ribosome protection (Roberts, 2005).

Table.1 Demographic data and microscopic examination of the studied samples $(\mathrm{n}=47)$. Values are number $(\%)$ or mean \pm SD (range).

\begin{tabular}{|l|c|c|l|lc|}
\hline & $\begin{array}{c}\text { Urine } \\
(\mathbf{n = 2 8})\end{array}$ & $\begin{array}{c}\text { HVS } \\
(\mathbf{n = 1 9})\end{array}$ & P- value & \multicolumn{2}{|c|}{$\begin{array}{c}\text { 95 \% confidence } \\
\text { interval } \\
\text { Lower }\end{array}$} \\
\hline Agp (y) & $\begin{array}{c}39.25 \pm 11.3 \\
(20-63)\end{array}$ & $\begin{array}{c}38.9 \pm 9.9 \\
(23-65)\end{array}$ & 0.9 & -6.1 & 6.8 \\
\hline WBC/HPF & $\begin{array}{c}85.96 \pm 315 \\
(1-1600)\end{array}$ & $\begin{array}{c}15.36 \pm 28.7 \\
(1-100)\end{array}$ & 0.3 & -75.8 & 217 \\
\hline RBCs/HPF & $\begin{array}{c}5.89 \pm 14.4 \\
(1-77)\end{array}$ & $\begin{array}{c}1.63 \pm 0.76 \\
(1-3)\end{array}$ & 0.2 & -2.4 & 10.9 \\
\hline PUS : & $\begin{array}{c}7(25 \%) \\
\text { High } \\
\text { Low }\end{array}$ & $\begin{array}{c}6(31.6 \%) \\
13(68.4 \%)\end{array}$ & 0.4 & -0.21 & 0.29 \\
\hline
\end{tabular}

HVS: High vaginal swab HPF: High power field
RBCs: Red blood cells

WBCs: White blood cells

Table.2 Frequency of group B Streptococci in different months ( $\mathrm{n}=47$ ).

\begin{tabular}{|l|c|c|c|c|}
\hline \multirow{2}{*}{ Month } & \multicolumn{2}{|c|}{ Urine sample (n=28) } & \multicolumn{2}{c|}{ HVS (n=19) } \\
\cline { 2 - 5 } & $\mathrm{N}$ & $\%$ & $\mathrm{~N}$ & $\%$ \\
\hline January & 0 & 0 & 1 & 5.3 \\
\hline February & 2 & 7.1 & 1 & 5.3 \\
\hline March & 1 & 3.6 & 2 & 10.5 \\
\hline April & 1 & 3.6 & 2 & 10.5 \\
\hline June & 1 & 3.6 & 4 & 21.0 \\
\hline July & 3 & 10.7 & 0 & 0 \\
\hline August & 2 & 7.1 & 1 & 5.3 \\
\hline September & 1 & 3.6 & 1 & 5.3 \\
\hline October & 7 & 25 & 3 & 15.8 \\
\hline November & 6 & 21.4 & 3 & 15.8 \\
\hline December & 4 & 14.3 & 1 & 5.3 \\
\hline Total & 28 & 100 & 19 & 100 \\
\hline
\end{tabular}

HVS: High vaginal swab 
Table.3 Seasonal variation in each group of UTI and vaginal infections.

\begin{tabular}{|l|c|c|c|c|c|}
\hline \multirow{2}{*}{} & \multicolumn{2}{|c|}{ Urine sample $(\mathrm{n}=28)$} & \multicolumn{2}{c|}{ HVS $(\mathrm{n}=19)$} & \multirow{2}{*}{ P-value } \\
\cline { 2 - 5 } & $\mathrm{N}$ & $\%$ & $\mathrm{~N}$ & $\%$ & \\
\hline Winter & 6 & 21.4 & 3 & 15.8 & \\
\hline Spring & 2 & 7.0 & 4 & 21.1 & \multirow{2}{*}{0.5} \\
\hline Summer & 6 & 21.4 & 5 & 26.3 & \\
\hline Autumn & 14 & 50 & 7 & 36.8 & \\
\hline Total & 28 & 100 & 19 & 100 & \\
\hline
\end{tabular}

HVS: High vaginal swab

Table.4 Frequency of Colony count in UTI with WBCs /HPF ( $\mathrm{n}=28$ ).

\begin{tabular}{|l|c|c|c|}
\hline & \multicolumn{2}{|c|}{ Colony count } & \multirow{2}{*}{ p-value } \\
\cline { 2 - 3 } & $10^{4}-10^{5}$ & $>10^{5}$ & \multirow{2}{*}{0.4} \\
\cline { 1 - 3 } $\begin{array}{l}\text { Low WBCs }(<10 / \mathrm{HPF}) \\
(\mathrm{n}=21)\end{array}$ & $9(42.9)$ & $12(57.1)$ & \\
\cline { 1 - 3 } $\begin{array}{l}\text { High WBCs }(\geq 10 / \mathrm{HPF}) \\
(\mathrm{n}=7)\end{array}$ & $1(14.3)$ & $6(85.7)$ & \\
\hline Total $(\mathrm{n}=28)$ & $10(35.7)$ & $18(64.3)$ & \\
\hline
\end{tabular}

Table.5 Relation of inducible clindamycin resistance, Erythromycin and Clindamycin susceptibility among GBS $(\mathrm{n}=47)$. Values are number $(\%)$.

\begin{tabular}{|l|c|c|c|c|c|c|c|}
\hline \multirow{2}{*}{$\begin{array}{l}\text { Inducible } \\
\text { Clindamycin }\end{array}$} & \multicolumn{3}{|c|}{ Erythromycin Susceptibility } & \multirow{2}{*}{$\mathrm{P}$} & \multicolumn{2}{|c|}{$\begin{array}{c}\text { ClindamycinSusceptib } \\
\text { ility }\end{array}$} & \multirow{2}{*}{$\mathrm{P}$} \\
\cline { 2 - 3 } & $\mathrm{S}$ & $\mathrm{R}$ & $\mathrm{I}$ & & $\mathrm{S}$ & $\mathrm{R}$ & \\
\hline Urine samples & & & & & & & \\
Negative (15) & $11(73.4)$ & $2(13.3)$ & $2(13.3)$ & & $12(80)$ & $3(20)$ & \\
Positive (13) & $0(0)$ & $13(100)$ & $0(0)$ & 0.000 & $0(0)$ & $13(100)$ & 0.000 \\
Total (28) & $11(39.3)$ & $15(53.6)$ & $2(7.1)$ & & $12(42.9)$ & $16(57.1)$ & \\
\hline HVS & & & & & & & \\
Negative (9) & $6(66.7)$ & $3(33.3)$ & $0(0)$ & & $7(77.8)$ & $2(22.2)$ & \\
Positive (10) & $0(0)$ & $10(100)$ & $0(0)$ & 0.003 & 0.0 & $10(100)$ & 0.001 \\
Total (19) & $6(31.6)$ & $13(68.4)$ & $0(0)$ & & $7(36.8)$ & $12(63.2)$ & \\
& & & & & & & \\
\hline
\end{tabular}

HVS: High vaginal swab 
Table.6 Antimicrobial susceptibility pattern of GBS $(n=42)$. Values are numbers $(\%)$.

\begin{tabular}{|l|c|c|c|c|c|c|}
\hline & \multicolumn{2}{|c|}{ Urine samples (n=28) } & \multicolumn{3}{c|}{ HVS (n=19) } \\
\cline { 2 - 7 } & $\mathrm{S}$ & $\mathrm{R}$ & $\mathrm{I}$ & $\mathrm{S}$ & $\mathrm{R}$ & $\mathrm{I}$ \\
\hline Ampicillin & $28(100)$ & 0 & 0 & $19(100)$ & 0 & 0 \\
\hline Benzyl penicillin & $28(100)$ & 0 & 0 & $19(100)$ & 0 & 0 \\
\hline Cefotaxime & $28(100$ & 0 & 0 & $19(100)$ & 0 & 0 \\
\hline Ceftriaxone & $28(100)$ & 0 & 0 & $19(100)$ & 0 & 0 \\
\hline Clindamycin & $\begin{array}{c}12 \\
(42.9)\end{array}$ & $\begin{array}{c}16 \\
(57.1)\end{array}$ & 0 & $\begin{array}{c}17 \\
(36.8)\end{array}$ & $\begin{array}{c}12 \\
(63.2)\end{array}$ & 0 \\
\hline Erythromycin & $\begin{array}{c}11 \\
(39.3)\end{array}$ & $\begin{array}{c}15 \\
(53.6)\end{array}$ & $2(7.1)$ & $6(31.6)$ & $\begin{array}{c}13 \\
(68.4)\end{array}$ & 0 \\
\hline Levofloxacin & $\begin{array}{c}23 \\
(82.1)\end{array}$ & $5(17.9)$ & 0 & $\begin{array}{c}15 \\
(78.9)\end{array}$ & $\begin{array}{c}4(21.1) \\
0\end{array}$ \\
\hline Linezolid & $28(100)$ & 0 & 0 & $19(100)$ & 0 & 0 \\
\hline Tetracycline & $1(3.6)$ & $\begin{array}{c}27 \\
(96.4)\end{array}$ & 0 & $1(5.3)$ & $\begin{array}{c}18 \\
(94.7)\end{array}$ & 0 \\
\hline TMP/SMZ & $\begin{array}{c}27 \\
(96.4)\end{array}$ & $1(3.6)$ & 0 & $19(100)$ & 0 & 0 \\
\hline Vancomycin & $28(100)$ & 0 & 0 & $19(100)$ & 0 & 0 \\
\hline
\end{tabular}

$\mathrm{S}=$ sensitive $\mathrm{R}=$ Resistant $\quad \mathrm{I}=$ Intermediate TMP/SMZ:Trimethoprim/Sulfamethoxazole HVS: High vaginal swab

In present study, GBS was highly susceptible to Ampicillin, Penicillin, Cefotaxime, Ceftriaxone, Linezolid, TMP/SMZ and Vancomycin.Woods, 2015 reported that GBS are uniformly sensitive to Penicillin and Ampicillin. Although resistance to Penicillin or Ampicillin has not be documented, some isolated have shown MIC approaching the upper limits of susceptibility for some of the Beta-Lactam agents (Phares et al., 2008).

Many studies reported high rate of susceptibility to Penicillin G (Koet al., 2001; Blancas et al., 2004; Huang et al., 2006). Shayanfar et al., 2012 showed that GBS was sensitive to Cephalothin, Norfloxacin, Ampicillin, Nitrofurantoin, Vancomycin, therefore treatment with Ampicillin or Cephalothin was recommended. Also, Chaiwarith et al., 2011, founded that all isolates of GBS were susceptible to
Penicillin, Ampicillin and Vancomycin. However, Rahbar et al., 2012 showed high resistance to Penicillin, Ampicillin, Ceftriaxone, Cefotaxime (89.4\%, 52.5\%, $43,8 \%, 46.6 \%)$. Also, in a study performed at Taiwan founded susceptibility to Penicillin $60.7 \%$ and $100 \%$ for Vancomycin (Fu et al., 2004).

As it is obvious from the results of these above reports, antibiotic resistance rates showed different pattern so, it indicates that performance of antibiotic susceptibility test is necessary before any prescriptions (Rahbar et al., 2012).

From this study we concluded that we must do culture if the patient complaining of manifestation of UTI even in presence of low WBCs count (absence of pyuria). Physicians can start empirically with Penicillin or Ampicillin for GBS but the 
problem in allergic patients to Penicillin so, we must do the antibiotic susceptibility due to the high resistance rate to Clindamycin and Erythromycin nowadays.

\section{References}

Blancas, D., Santin, M., Olmo M., et al. 2004. Group B streptococcal disease in nonpregnant adults: incidence, clinical characteristics and outcome. Eur. J. Clin. Microbiol. Infect. Dis., 23: 168-73.

Bliss, S.J., Manning, S.D., Tallman, P., et al. 2002. Group B Streptococcus colonization in male and non-pregnant female university students: a crosssectional prevalence study. Clin Infect Dis., 34(2):184-90.

Bowling, J.E., Owens, A.E., McElmeel, M.L., et al. 2010. Detection of inducible clindamycin resistance in BetaHemolytic streptococci by using the CLSI broth microdilution test and erythromycin-clindamycin combinations. J. Clin. Microbiol., 48(6): 2275-2277.

Chaiwarith, R., Jullaket, W., Bunchoo, M., et al. 2011. Streptococcus agalactiae in adults at Chiang mai University hospital: a retrospective study. BMC Infect. Dis., 11: 149-156.

Domelier, A.s., Van, dM.M., Arnault, L., et al. 2008. Molecular characterization of erythromycin- resistant Streptococcus agalactiae strains. J. Antimicrob. Chemother., 62: 1227-33.

Edwards, M.S., and Baker, C.J. 2005. Group B streptococcal infections in elderly adults. Clin. Infect. Dis., 41: 839-847.

Edwards, M.S., Rench, M.A., Palazzi, D.L., Baker, C.J. 2005. Group B streptococcal colonization and serotype-specific immunity in healthy elderly persons. Clin. Infect. Dis., 40(3): 352-7.

Falagas, M.E., Rosmarakis, E.S., Avramopoulos, I., Vakalis, N. 2006. Streptococcus agalactiae infections in non-pregnant adults: single center experience of a growing clinical problem. Med. Sci. Monit., 12(11): 44751.

Farley, M.M., Harvey, R.C., Stull, T., et al. 1993. A population- based assessment of invasive disease due to group B streptococcus in non-pregnant adults. $N$. Engl. J. Med., 328: 1807-11.

Florindo, C., Damiao, V., Silvestre, I., et al. 2014. Epidemiological surveillance of colonizing group B Streptococcus epidemiology in the Lisbon and Tagus Valley regions, Portugal (2005 to 2012): emergence of a new epidemic type IV/clonal complex 17 clone. Euro Surveill., 19(23): pii=20825.

Fu, J.C., Lin, D.P., Huang, W.J., et al. 2004. Antibiotic susceptibility pattern of anovaginal isolates of Streptococcus agalactiae from pregnant women in their late third trimester. The Kaohsiung J. Med. Sci., 20(7): 330-3.

Gherardi, G., Imperi, M., Baldassarri, L., et al. 2007. Molecular epidemiology and distribution of serotypes, surface proteins and antibiotic resistance among group B streptococci in Italy. J. Clin. Microbiol., 45(9): 2909-16.

Gosnell, C.M., Fortune, V., Yu, C., et al. 2005. Detection of Clindamycin and Erythromycin Resistance in Isolates of Streptococcus agalactiae using Three Commercial systems. As presented at the $105^{\text {th }}$ General Meeting of the American Society for Microbiology.

Gygax, S.E., Schuyler, J.A., Kimmel, L.E., et al. 2006. Erythromycin and clindamycin resistance in group B streptococcal clinical isolates. Antimicrob. Agents Chemother., 50:1875-7.

Huang, P.Y., Lee, M.H., Yang, C.C., Leu, H.S. 2006. Group B streptococcal bacteremia in non-pregnant adults. $J$. Microbiol. Immunol. Infect., 39: 237-41.

Ko, W.C., lee, H.C., Wang, L.R., et al. 2001. Serotyping and antimicrobial susceptibility of group B Streptococcus over and eight-year period in southern Taiwan. Eur. J. Clin. Microbiol. Infect. 
Dis., 20: 334-9.

Lambiase, A., Agangi, A., Del Pezzo, M., et al. 2012. In vitro resistance to Macrolides and Clindamycin by Group B Streptococcus isolated from pregnant and non- pregnant women. Infectious Diseases in Obstetrics and Gynecology. Article ID 913603, 5 pages. Doi:10.1155/2012/913603.

Mandell, G.L. 2000. Mandell, Douglas and Benett's Principles and practice of infectious diseases. $5^{\text {th }}$ edn. New York: Churchill-Livingstone, 2156-64.

Matsubara, K., Yamamoto, G. 2009. Invasive group B streptococcal infections in a tertiary care hospital between 1998 and 2007 in Japan. Int. J. Infect. Dis., 13: 679-84.

Matteson, K.A., Lievense, S.P., Catanzaro, B., Phipps, M.G. 2008. Intrapartum group B streptococci prophylaxis in patients reporting a penicillin allergy. Obstet. Gynecol., 111: 356-64.

Mckenna, D.S., Matson, S., and Northern, I. 2003. Maternal group B streptococcal (GBS) genital tract colonization at term in women who have asymptomatic GBS bacteriuria. Infect. Dis. Obstet. Gynecol., 11: 203-207.

Munoz, P., Coque, T., Rodriguez Creixems. M., et al. 1992. Group B streptococcus: a cause of urinary tract infection in nonpregnant adults. Clin. Infect. Dis., 4: 492-6.

Nolla, J.M., Gomez-Vaquero, C., Corbella, X., et al. 2003. Group B Streptococcus (Streptococcus agalactiae) pyogenic arthritis in non-pregnant adults. Medi., 82(2): 119-128.

Phares, C.R., Lynfield, R., Farley, M.M., et al.
2008. Epidemiology of invasive group B streptococcal disease in the United States, 1999-2005. JAMA, 299: 205665.

Rahbar, M., Hajia, M., Mohammadzadeh, M. 2012. Urinary Tract Infections caused by group B Streptococcus in adult women: Survey of 11800 urine culture results. Iranian J. Pathol., 7(1): 32-37.

Roberts, M.C. 2005. Update on acquired tetracycline resistance genes. FEMS Microbiol. Lett., 245: 195-203.

Schuchat, A. 1999. Group B streptococcus, Lancet, 353(9146): 51-56.

Shayanfar, N., Mohammadpour, M., HashemiMoghadam, S.A., et al. 2012. Group B Sreptococci urine isolates and their antimicrobial susceptibility profiles in a group of Iranian females: Prevalence and seasonal variations. Acta Clin. Croat., 51: 623-626.

Tazi, A., Reglier-Poupet, H., Raymond, J., et al. 2007. Comparative evaluation of VITEK 2 for antimicrobial susceptibility testing of group B Streptococcus. J. Antimicrobiol. Chemother., 59: 1109-1113.

Toumi, A., Ferjani, A., Ben Abdallah, H., Boukadida, J. 2006. Streptococcus agalactiae in non-pregnant adults. Tunis Med., 84(3): 161-4.

Ulett, K.B., Benjamin, W.H., Zhuo F., et al. 2009. Diversity of Group B Streptococcus serotypes causing urinary tract infection in adults. J. Clin. Microbiol., 2055-2060.

Woods, C.J. 2015. Streptococcus Group B Infections treatment and management. Medscape.

\section{How to cite this article:}

Mona F. Foad. 2016. Urinary Tract and Vaginal Infections caused by Group B Streptococcus and the Macrolide-Inducible Resistance to Clindamycin in Non-pregnant Females. Int.J.Curr.Microbiol.App.Sci. 5(8): 486-496. doi: http://dx.doi.org/10.20546/ijcmas.2016.508.052 